\title{
LHomme
}

L'HOMME Revue française d'anthropologie

$190 \mid 2009$

Varia

\section{Secrets et organisation politique kanake}

Pour sortir des catégories privé/public

. Moving Beyond the Categories of "Private" and "Public"

\section{Christine Demmer}

\section{(2) OpenEdition \\ Journals}

Édition électronique

URL : http://journals.openedition.org/lhomme/22105

DOI : 10.4000//homme.22105

ISSN : 1953-8103

Éditeur

Éditions de l'EHESS

Édition imprimée

Date de publication : 1 janvier 2009

Pagination : 79-104

ISSN : 0439-4216

Référence électronique

Christine Demmer, « Secrets et organisation politique kanake », L'Homme [En ligne], 190 | 2009, mis en ligne le 01 janvier 2011, consulté le 01 mai 2019. URL : http://journals.openedition.org//homme/22105 ; DOI : 10.4000/lhomme.22105 


\title{
Secrets et organisation politique kanake \\ Pour sortir des catégories privé/public
}

\author{
Christine Demmer
}

$I_{\mathrm{L}}$

L EST FRAPPANT de constater que les premiers observateurs occidentaux du monde kanak qui se sont essayés à décrire les systèmes politiques de la Grande Terre et des îles aient proposé deux visions pour le moins opposées de la chefferie : entre une image positive d'un chef peu présent et sans pouvoir et une autre figure beaucoup plus inquiétante d'un chef autoritaire, voire sanguinaire. Les recherches menées depuis les années 1950 sur les chefferies (ces espaces de résidence qui sont aussi des espaces politiques hiérarchisés et centralisés) laissent entendre que ces divergences ne peuvent tenir à des variations locales qui, bien que réelles, n’en sont pas pour autant aussi radicales. De même, la possible confusion chez ces premiers observateurs entre chef et maître de la terre (tous deux de haut rang et identifiables comme " chefs") ne peut conduire à de telles différences d'interprétations. Il me semble qu'il faut plutôt chercher la réponse du côté de la difficulté à cerner ce qui sépare sphère privée et sphère publique dans ces univers politiques. Pour avancer sur ces questions, je me propose d'interroger l'usage du secret au sein des chefferies, car ce dernier a un rôle à jouer dans la structuration de ces espaces politiques. Plus précisément, je défends l'idée que la manière dont sont manipulés les secrets souligne le caractère problématique d'une catégorisation des composantes sociales d'une chefferie en termes de sphère privée d'un côté et sphère publique de l'autre. L'introduction de l'ouvrage d'Habermas sur l'espace 
public bourgeois ${ }^{1}$ - qui évoque à cet endroit le modèle féodal - a alimenté ma réflexion. Pour décrire les univers politiques de l'Europe du Moyen Âge, il fait en effet plutôt valoir les catégories de commun et de particulier : le premier qualificatif est donné à l'homme "ordinaire» et le second à celui qui possède des privilèges, base d'un pouvoir et donc de ce qui pourrait être désigné comme public dans le modèle du droit romain ${ }^{2}$. Bien que je ne considère en aucune façon le modèle politique kanak comme une réplique ou même un avatar de celui féodal, j’ai tâché néanmoins de réfléchir à l'emploi des catégories qu'Habermas attache à ce dernier pour décrire la structuration des espaces politiques kanaks. Dans la mesure où les catégories de privé et public ne me semblent pas adaptées, celles de commun et de particulier méritent, à mon sens, qu'on y prête attention. Sans trop dévoiler de mon argumentaire à suivre, je peux d'ores et déjà avancer qu'elles permettent de souligner ce qui me paraît partagé par ces deux systèmes hiérarchisés : à savoir l'asymétrie de position sociale entre les personnes que l'on range dans l'une et l'autre sphères et, surtout, l'existence d'une certaine continuité entre ces sphères ${ }^{3}$.

Pour décrire le modèle kanak, je prendrai appui sur deux formes de secrets. Les premiers sont relatifs à la fondation d'une chefferie. Ils nous apprennent quelque chose du modèle politique, contribuant à distinguer les clans de haut rang des clans de rang moindre et à rendre complémentaires tous les clans entre eux. Les seconds - gardés plus ou moins précieusement au sein des clans, dissimulés pour partie lors des discours relatifs au groupe politique local énoncés sur une place commune ou devant une allée cérémonielle - introduisent à la dynamique politique. Ils permettent de réactiver/modifier un équilibre hiérarchique structurellement instable. Si l'ensemble de ces secrets revêt un contenu politique, certains d'entre eux sont spécifiques aux personnalités importantes d'une tribu, d'autres sont propres aux unités segmentaires patrilinéaires, d'autres encore sont communs à la totalité du groupe de résidence.

Mon propos reposera sur des enquêtes de terrain réalisées dans la commune de Canala (aire linguistique xârâcùù) sur la Grande Terre. Avant d'en venir à l'usage des secrets au sein des espaces de résidence, je commencerai par rappeler les formes d'organisation des chefferies de

1. «Définition propédeutique d'un modèle de la sphère politique bourgeoise " (in Habermas 1978: 13-38).

2. Définitions à ne pas confondre avec celles données par Habermas (1978: 18), où l'ancienne juridiction germanique, à la différence de la période féodale, établissait une correspondance entre « commun » et "public " (parce qu'accessibles à tous), et "particulier » et "privé " (parce que séparés).

3. Ces caractéristiques, Habermas les confere à ce qu’il nomme «la sphère publique structurée par la représentation" (1978: 17). 
la commune pour souligner les difficultés de catégorisation au regard des termes privé/public. Ces chefferies comportent de nombreux points communs avec celles d'autres aires politiques de la Grande Terre néocalédonienne ou des îles Loyauté mais sont, à divers titres, originales - ce qui justifie de préciser dans quels lieux s'ancrent mes descriptions.

\section{Introduction à l'organisation politique kanake Un espace politique original au sein de la République}

Le temps de l'indigénat

Sous leur forme actuelle, les villages de Canala sont, comme les autres villages, le fruit du cantonnement opéré tout au long de la seconde moitié du XIX ${ }^{e}$ siècle. Ils s'inscrivirent dans la catégorie administrative coloniale de "la tribu», personne morale reconnue responsable et propriétaire des terres. Ce cantonnement a établi pour les Kanaks non seulement un statut foncier à part (des terres incommutables, insaisissables et inaliénables), mais il leur a conféré aussi un statut personnel différent (statut civil particulier qui consacra leur assujettissement, notamment par l'instauration en 1887 d'un Code de l'indigénat limitant leur possibilité de travailler et circuler librement et qui les soumettait à un impôt de capitation). Parallèlement à l'enfermement dans les réserves, l'État français a mis en place une administration indirecte, reconnaissant des "chefs" (qui à Canala se nomment aaxa, "ceux qui parlent») ayant autorité sur des "sujets" (kwara). Ces personnages étaient redevables des atteintes à l'ordre public colonial au sein des villages et chargés de fournir de la main-d'œuvre et de récolter l'impôt de capitation (Lenormand 1953; Merle 1998 ; Soriano 2001). Les villages de réserve, sont, encore aujourd'hui, des entités politiques nommées "chefferies" (nèaaxa rè à Canala, "l'espace de ceux qui

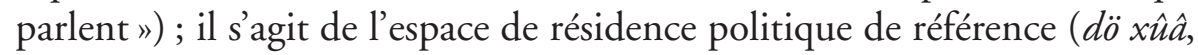
«maison authentique»). Tous mes interlocuteurs l'ont souligné, sans être la copie conforme des systèmes politiques précoloniaux, les nèaaxa rè actuels en reprennent nombre de références passées. Ces chefferies, notamment, s'appuient toujours sur une hiérarchie statutaire distinguant des clans sujets et des clans plus nobles. De ce point de vue, l'organisation sociale de Canala renvoie, dans ses grandes lignes, à celle des autres aires kanakes : logique segmentaire, centralisation politique et hiérarchisation selon un principe de primorésidence ${ }^{4}$. 
Dans cette commune, les petites chefferies villageoises sont au nombre d'une vingtaine réparties en quatre grandes chefferies. Emma (Amââ) dont il va être question s’inscrit dans les limites de la grande chefferie Kèrèduru - regroupant sans conteste quatre villages (sept selon certaines versions recueillies au sujet de l'histoire régionale). Ces grandes chefferies sont toutes inscrites dans le périmètre de réserves, globalement soumises à l'État français. Elles forment deux «districts» administratifs : le plus important à Canala se nomme Bwaaxéa (dont dépend Kèrèduru) et l'autre Nôôme (dont dépend la grande chefferie de Penyî mê Nèkètè [Nakéty]).

\section{L'après Seconde Guerre mondiale}

L'inscription de ce monde hiérarchisé dans l'espace politique national français s'est réalisée à la marge dès les premières décennies de la colonisation. Mais l'abolition du Code de l'indigénat en 1946 a marqué le point de départ d'une nouvelle articulation entre pouvoir global et pouvoir local. Les Kanaks, toujours en réserves, sont devenus citoyens de "droits particuliers " (ils ont acquis le droit de vote en 1951). L'État s'étant opposé à l'institutionnalisation de l'autorité des chefs a admis toutefois la spécificité politique d'un tel pouvoir dit " coutumier». Il a tenté de renforcer une assemblée politique, repérée apparemment par les premiers observateurs du monde kanak, légitimée et transformée par le système colonial: le "conseil des anciens". Ce dernier est composé soit des personnages importants d'une chefferie, soit des aînés des clans présents. Après 1946, même si le droit commun français prime sur le droit coutumier, les tribus, par leurs chefs et, selon les cas, leurs conseils des anciens, ont conservé des prérogatives particulières soustraites au domaine du droit public (en matière foncière, successorale, ainsi que dans le régime d'adoption et, jusqu'en 1967, pour les mariages). Dans la pratique, les chefferies se réservent encore le règlement de certains délits.

La tribu demeure donc un espace régi par des normes particulières au regard de la loi française comme du point de vue de l'organisation interne au monde politique kanak. Les chefferies kanakes sont, à présent, des espaces politiques qui ne sont ni totalement autonomes ni figés sur un modèle précolonial, mais qui n’en demeurent pas moins des entités politiques spécifiques.

\section{Les principales références de l'organisation politique}

Logique segmentaire (des groupes semi-publics)

Toutes les chefferies kanakes sont composées de regroupements d'unités segmentaires. Celles de Canala ne font pas exception. Cette commune ayant été peu étudiée du point de vue de son organisation sociale avant mon passage à la fin des années 1990, ce qui suit relève d'un travail de mise 
à plat effectué par mes soins au début de mes enquêtes de terrain ${ }^{5}$. Le terme de xwâmwââdö, "clan " en langue xârâcùù, signifie littéralement "l'ouverture» (xwâ) de la "grande case» (mwââä̈). Il sert à désigner quasiment tous les niveaux de la segmentation des unités de filiation patrilinéaire qui donnent corps aux chefferies. On peut les distinguer, des plus grandes au plus restreintes, en utilisant les termes suivants: grands clans, clans, lignages en binôme, lignages simples et lignées ${ }^{6}$. Les Kanaks utilisent pour leur part, en français, le terme de clan pour toutes ces unités sociales, à l'exception des deux dernières appelées "familles ». Les individus appartenant à un même xwâmwââdö se disent liés par le sang mais, comme les termes vernaculaires l'indiquent, c'est la corésidence qui les apparente à un "aîné ». Ils se rattachent à un fondateur de tertre (mê mwâ) autant qu'à ce tertre lui-même. C'est à partir de ce tertre qu'ils tirent nom et jouissance d'un espace foncier proche. Ce modèle simplifié d'appartenance est, en réalité, beaucoup plus complexe. Les groupes et individus de cette aire linguistique, comme tous les autres, se sont déplacés avant la colonisation et aussi tout au long du cantonnement (Barrau 1956; Saussol 1979; Dauphiné 1989). Comme l'attestent aussi bien les récits que l'on peut recueillir que les archives, les mouvements de population les plus importants à Canala débutent dans les années 1850 et 1960 pour culminer avec la grande révolte kanake de 1878 (Dauphiné 1990). Les membres d'un groupe local peuvent ainsi, en remontant dans leur généalogie, faire valoir nombre d'appartenances en se revendiquant de diverses unités de filiation.

Mais il importe surtout de retenir à ce stade que le terme de "grande case » contenu dans le concept kanak «clan » réfere à un espace clos - si ce n'est domestique -, qu'on est tenté d'identifier à un espace privé. Cela semble confirmé par le fait que chaque xwâmwââdö, à tous les niveaux de la segmentation, se reconnaît avant tout par le partage d'une même histoire généalogique (xwâânîmö) qui, en principe, doit se transmettre uniquement au sein du groupe d'agnats. Ce type de récit - tenu secret consigne le lieu de naissance et le nom de l'ancêtre fondateur. Il décrit aussi la manière dont ce dernier s'est implanté dans une tribu au cours du cantonnement (l'éventuelle dation d'un nouveau nom lors de son arrivée ou l'éviction d'anciens clans par faits de guerre). Entre les deux moments, ce récit évoque les causes des déplacements voulus ou contraints, ainsi que

5. Il existe deux écrits antérieurs traitant de l'organisation sociale xârâcùù : celui de Jean Guiart (1962) et celui de Jean-Pierre Doumenge (1975).

6. À noter que la lignée porte un nom kanak spécifique : xwâjöösé («ceux issus de la grande corbeille du toit de la case»). La corbeille rassemble tous les chevrons autour du poteau central dans une maison traditionnelle, elle marque donc métaphoriquement l'unité de ceux qui font partie de la "case ", c'est-à-dire, dans ce contexte, qui sont issus d'un même ancêtre en ligne directe. 
les étapes du trajet parcouru, jalonné de toponymes, d'alliances et de combats. Il s'agit, à travers ces récits, de retracer l'identité sociale des groupes et leur place dans le dispositif politique local - voire dans les espaces de résidences antérieurs - sur le principe d'une classification par ordre d'ancienneté marqué par l'attachement à des tertres précis ${ }^{7}$.

Le caractère privatif des groupes agnatiques semble renforcé à Canala au niveau de la lignée (xwâjöösé); la forte prise en compte des lignes utérines rend ici irréductible l'assimilation des familles nucléaires entre elles (à la condition, bien évidemment, que les femmes de frères soient originaires d'un xwâmwââdö différent) ${ }^{8}$. La femme apporte avec elle certains savoirs propres à son clan et donne naissance à des enfants dont l'enveloppe corporelle sera considérée comme distincte de celle des enfants de frères ${ }^{9}$. On dit que chacune des maisonnées «a un morceau d'histoire différente à raconter " du fait de l'alliance (xwâxûâ bwètèe -ru ${ }^{10}$. Le degré d'imbrication des deux lignes agnatiques intermariées est rendu encore plus marquant en raison du système de parenté propre à Canala : de type Omaha, il infléchit la distinction entre parents et alliés ${ }^{11}$. Ces spécificités des rapports de parenté sont peut-être à l'origine d'une perception des maisonnées (xwâjöösé) moins en tant qu'associations de deux groupes privés qu'en tant qu'unités conjugales elles-mêmes privatives. Cela peut s'entendre à travers l'expression qu'on utilise parfois pour les désigner : " ceux qui mangent dans la même marmite " (êrê döchaa kùrè) et, pour les enfants, "gens issus d'un même ventre" (êrê döchaa pwè).

Ce qui vient d'être exposé n'empêche en rien de considérer que les alliances - comme les adoptions d'ailleurs - ont une dimension politique très forte. Elles servent avant tout à créer et/ou à renouveler au fil du temps, par leur réitération, les relations entre deux clans ${ }^{12}$. Celles-ci sont

7. Sur le rôle des récits historiques dans la constitution des statuts sociaux kanaks, voir Bensa \& Rivierre (1982) et Naepels (1998).

8. On ne manque jamais de relever les liens de fraternité ( $p a$ bé) entre deux individus issus de grands-mères qui sont sœurs et l'on figure la ligne utérine par la référence à une liane donnant de nombreuses ramifications à partir du pied originel.

9. Dans les discours politiques, la vie d'une personne, tout ce qui constitue l'enveloppe corporelle et ce qui l'anime, est attribuée aux lignes utérines. Pour la constitution de la personne, voir Bensa \& Goromido (1998) et Salomon (1998).

10. Xwâxûâ, c'est l'" ouverture de la maison ", bwètèè signifie "bout, partie».

11. Dans la terminologie de parenté, la tante paternelle est en quelque sorte l'égale de son neveu dans la mesure où ils s’autodésignent par le même terme «chää» alors même que cette tante représente le groupe potentiellement allié de celui d'ego. De même, l'oncle maternel se trouve sur un plan d'équivalence avec les grands-pères à cause de l'emploi oblique du terme "xèrè " qui signifie aussi bien neveu/nièce que petit-fils/petite-fille.

12. On dit que les lignes utérines sont menacées lorsque deux frères se disputent : «ils marchent sur le feu des tontons ". Référence est faite à l'image - répandue - considérant le foyer culinaire comme le fruit de l'association de deux clans. 
activées ensuite chaque fois que nécessaire pour faire face à des problèmes précis (de l'ordre de l'intime, mais aussi, lors de conflits ayant trait à la compétition statutaire). Dans les cérémonies de mariage, le clan donneur de femme qui s'adresse au preneur insistera sur le rôle de la femme en tant que "celle qui est à l'origine de la lignée" (puudèeri), mais dira aussi que la femme vient "procréer pour la prospérité du pays " (xërù jati mwâciri). Si la femme "fait vivre" une unité segmentaire, elle fait donc aussi, au-delà, "vivre " une unité politique. Un effectif démographique important est indispensable aussi bien à l'établissement qu'à la pérennisation de la puissance d'une chefferie. Autrefois, il garantissait la force guerrière ; aujourd'hui encore, il s'avère support nécessaire à la captation de la force des ancêtres à travers les reliques (Bensa 1992).

La puissance numérique se conjugue, pour certains, à un rang élevé. C'est le nom du clan qui exprime le rang d'un individu; en tant que tel, il inscrit le groupe agnatique dans un dispositif politique. Les récits généalogiques (xwâânîmö) recueillis à Canala relatifs à la période du cantonnement montrent que l'arrivant pouvait être adopté dans le clan qui l'accueillait et prendre le nom de l'un des lignages de ce clan. De même, avant la pacification, un guerrier victorieux pouvait s'octroyer la place d'aîné, éliminant ou rabaissant au rang de simples sujets les anciens occupants de ce titre. Un étranger de haut rang dans sa chefferie d'origine retrouvait, en se déplaçant, un rang important ailleurs. Il pouvait devenir chef à la place d'un autre. Enfin, il était exceptionnellement possible que l'accueilli soit assis à une place inédite. Quant aux gens d'un rang moindre, ils pouvaient intégrer d'autres clans sujets, prendre même le nom d'un groupe agnatique qui n'avait plus de membres. Même en bas de l'échelle, le nouvel arrivant participe toujours du dispositif politique local. L'accueil ( $f a-c u e ̀$, "faire asseoir ») signale donc, en même temps que l'intégration à un clan, l'attribution de titres nouveaux au sein d'une chefferie. Derrière l'énoncé de ces diverses modalités d'intégration au groupe politique - à l'exception de celle concernant l'éviction des fondateurs d'un lieu -, il faut bien comprendre que la place occupée au sein d'une chefferie (réinterprétée dans le cadre idéel du degré de proximité au fondateur du lieu) est éminemment dépendante de décisions politiques propres aux clans dits " autochtones ${ }^{13}$. En ce sens, l'identité sociale de tous les clans sans exception - dont l'assimilation à des groupes de parenté peut les ranger du côté de la catégorie de privé - relèverait plutôt d'un cadre semi-privé ou, si l'on

13. Les récits font état d'une formule rhétorique codifiée, "Toi tu vas "parler" [xa] pour ", qui signale le caractère quasi contractuel de l'acquisition d'un statut et de pouvoirs assimilés à une " parole » précise contre l'apport de compétences et de magies mises au service du groupe local. 
préfere, semi-public. J'en veux pour preuve la manière dont sont résolues

les affaires communes à un de ces groupes. Bien qu'à leur échelle ils soient en mesure de gérer certains de leurs problèmes (notamment fonciers) sans en référer aux autorités des autochtones, ils ne peuvent s'en tenir là si le conflit s'avère récurrent ou bien si le compromis trouvé est jugé insatisfaisant par l'un des membres du clan. En somme, l'autorité du clan est subordonnée à une autorité centralisatrice.

\section{Les "nobles" comme représentants de la sphère publique?}

Mes discussions avec des érudits kanaks m'ont permis de formuler certaines hypothèses concernant la chefferie précoloniale. Juste avant l'enfermement dans les réserves, l'espace résidentiel du grand clan (xwâmwââdö) aurait été borné uniquement par des repères topographiques importants (rivières, montagnes). Ce "pays" (mwâciri) aurait été jalonné d'allées ou des places (xwâjööchaa) correspondant à des tertres fondés successivement. L'allée principale du mwâciri aurait été celle du premier occupant de ce lieu, espace auparavant vierge ou abandonné; les autres allées auraient été celles des xwâmwââdö plus restreints et plus récents (clans, lignages doubles et simples) qui lui étaient apparentés. Plutôt que d'imaginer des limites territoriales continues, mes interlocuteurs insistent pour souligner qu'il faut envisager le "pays" d'alors comme l'étendue couvrant l'extension maximale des relations de dépendance établies au sein du même grand clan. Mais des unités segmentaires appartenant à d'autres grands clans pouvaient s'intercaler sur ce même terroir ; des liens d'alliance s'établissaient apparemment entre eux. Ce n'est qu'avec les opérations massives de spoliations foncières - de 1876 jusqu'en 1894 à Canala (Dauphiné 1990) - que les chefferies coloniales ont acquis progressivement une dimension territoriale plus forte; l'organisation politique est devenue dans le même mouvement polyclanique (une même chefferie se composant alors de membres d'anciens grands clans différents). On ne parlera donc plus désormais du grand clan pour désigner un nèaaxa rè, mais d'une réunion de clans autour d'un chef. Ce dernier n'est plus identifié comme étant l'aîné mais désigné comme un autochtone (parmi ceux qui l'ont fait asseoir). Tout se passe comme si, la référence à l'espace se renforçant, le vocabulaire de la parenté s'effaçait au profit de celui de la résidence. Chacun de ces nouveaux clans regroupe généralement deux lignages binômes ou simplement plusieurs lignages simples (ou "familles») ${ }^{14}$. Jusqu'en 1946 - en raison de l'impossibilité de circuler

14. On a aussi affaire à la fusion de lignages de grands clans différents, voire à la création de nouveaux noms de clans. 
librement -, l'intensification notoire des alliances au sein des mêmes villages renforcera les liens noués entre ces groupes agnatiques et leur chef. Pour autant, les membres de ces clans se revendiquent toujours, lors d'occasions cérémonielles surtout, de ces groupes de filiation antérieurs : des listes de grands clans composés de plusieurs lignages binômes sont déclamées qui renvoient à des configurations sociales antérieures propres à des "pays " (mwâciri) contemporains des débuts de la présence française, voire précoloniaux, parfois assez éloignés du lieu de vie actuel de leurs descendants.

À Canala, les limites de l'espace des chefferies issues du cantonnement sont symbolisées par ce que les gens nomment des "barrières" (be). En réalité, il s'agit de rochers; chacun est supposé matérialiser les clans présents et leurs attributs magicoreligieux spécifiques. Ces derniers étaient utiles au bon fonctionnement de la chefferie avant les années 1920 (c'està-dire avant l'évangélisation de la région) ; malgré le passage des missionnaires, ils n'ont pas totalement perdu d'effectivité ${ }^{15}$. Aussi considère-t-on ces rochers soit comme des guerriers, soit comme les parties d'un corps humain. L'une et l'autre image supposent que ces rochers servent aussi bien à défendre l'intégrité physique des membres du groupe qu'ils ne participent à sa reproduction vitale. Pour souligner le caractère complémentaire des unités segmentaires au sein de cette organisation politique, les gens de Canala, à l'instar des Kanaks de toutes les aires linguistiques, recourent encore à une métaphore architecturale: celle de la maison kanake avec son poteau central (le chef) et les poteaux du tour de la case (les clans sujets). Ce faisant, ils désignent leur espace public de référence par un symbole qui relève du domestique!

Au-delà de l'anecdote, l'affirmation très forte et récurrente de la complémentarité entre les clans (dans une logique d'autovalorisation liée aux revendications nationalistes) tend à occulter la dimension hiérarchique - pourtant fondamentale - de ce système politique. Les groupes agnatiques appartiennent soit à un rang chef (Aaxa) - idéellement ce sont les plus anciens - soit à un rang sujet (Kwara) - idéellement ce sont les plus récemment implantés dans un terroir ${ }^{16}$. En fait, les positions de haut rang

15. Voir le film documentaire d'Emilio Pacull \& Christine Demmer, Emma, tribu kanake aujourd'hui (La Sept, cop. 1998).

16. Dans la plus ancienne des configurations politiques décrites (grand clan), on dit que les individus étaient subordonnés, à chaque niveau de la segmentation, au fondateur du tertre auquel ils se rattachaient, jusqu’à faire allégeance au premier occupant. Ce dernier est présenté, du point de vue actuel, comme l'ancêtre du grand clan d'une étendue territoriale qui regroupait symboliquement toutes les xwâjööchaa ("allées résidentielles») autour de son allée. Aussi, la plupart du temps, mes interlocuteurs soulignent qu'avant la colonisation il n'y avait pas de chef d'un côté et de maîtres de la terre de l'autre, mais seulement ces "aînés ". Cependant, il faut penser que la distance temporelle aidait, et il devient malaisé de distinguer (comme on peut le faire encore pour des chefferies plus récentes) si ce " chef » figurait parmi les derniers arrivants et n'était, en réalité, qu’assimilé à un aîné. 
(Aaxa) se déclinent selon trois modes à Canala : le aaxa proprement dit

("celui qui parle», présenté comme accueilli par les autochtones et intégré à leur rang), les kwara aéé ("grands sujets", qui sont dits autochtones) encore rarement appelés apu döö (les "maitres de la terre") et le kwara bwatù ("sujet qui s'abaisse» ou "sujet direct», ligne cadette des autochtones). Les kwara aéé sont considérés comme gardiens des magies de la chefferie, gardiens de la terre (de ses ressources) et de l'intégrité territoriale du terroir. Le kwara bwatù, cadet des autochtones, remplit la fonction de gardien du chef; il est celui qui le protège directement, qui transmet des messages entre groupes et sert de "faiseur de paix». Pour toutes ces fonctions, on le nomme encore apèrè-tèpe (le "porte-parole") ou xwâ̂̀ tèpe (la "route de la parole»), voire kwara pîtùrù ("sujet qui cache» [le chef]). Il pouvait, autrefois, aller jusqu'au sacrifice de sa personne. La distinction parmi les gens de haut rang de ce kwara bwatù ("sujet qui s'abaisse») fait du système politique de Canala un système un peu particulier sur la Grande Terre où généralement les autochtones forment un seul et même bloc de "soutiens" de la chefferie (Bensa \& Goromido 1998). Le chef à un rôle plus symbolique. Il est le garant de l'unité et de la survie du groupe. En dehors - et avant même son rôle joué auprès de l'administration coloniale -, il est au sommet d'une organisation politique comme celle décrite par Hocart (1978). Il joue un rôle rituel en tant que coordonnateur des activités humaines et cosmiques. On estime en effet que c'est grâce à lui que les tâches accomplies par tous portent leurs fruits et permettent la reproduction du groupe (Bensa 1988). Il synthétise donc en quelque sorte, en sa personne, toutes les unités sociales présentes. Une telle capacité lui provient de sa proximité supposée avec les ancêtres. Accueilli et assimilé aux autochtones, il en devient le représentant vivant. Les « rites pour la vie» qu'on accomplissait autrefois étaient ici des « rites pour le chef ». On rejoint sur ce point le modèle féodal décrit par Habermas, qui " rend tangible, à travers la personne publiquement présente du seigneur, un être invisible " où une personne de haut rang "se désigne et se présente comme l'incarnation d'une autorité comme toujours "supérieure" " (1978 : 19). Aujourd'hui, la dimension rituelle de la chefferie s'estompe dans la pratique mais le principe du chef symbole et incarnation du groupe demeure. Les critiques relatives à sa vie intime peuvent toujours déstabiliser momentanément une organisation politique locale et l'on dit toujours «travailler pour le chef ${ }^{17}$. Ce dernier n'est donc pas mandaté

17. Ayant étudié, dans le cadre de ma thèse, un projet de développement assumé par l'ensemble des clans de la petite chefferie d'Emma - et initié en partie par son chef -, j'ai pu entendre à plusieurs reprises que le travail accompli l'était "pour le chef ». 
pour représenter ses sujets; ce qui est public est lié à sa personne concrète. Aussi cela laisse-t-il supposer que l'espace public kanak est en quelque sorte confisqué par le chef à son seul profit - privatisé pourrait-on dire par la figure du chef, derrière laquelle se tiennent ses «soutiens".

\section{Pour une autre catégorisation du politique}

L'inadéquation des catégories privé/public

Pour autant, comme on l'a vu, les unités agnatiques ne sont pas en dehors de l'espace public. Même au plus bas de l'échelle, un groupe est intégré de plein droit - par la procédure de l'accueil au sein d'un terroir à un dispositif politique fondé, certes sur l'inégalité des statuts, mais aussi sur la complémentarité des rôles. Les sujets (Kwara) peuvent se revendiquer une "filiation» avec le chef. Celle-ci les inclut dans cette hiérarchie englobante qui classe ses groupes en fonction du lien - même si ce dernier est le plus lointain - aux ancêtres fondateurs et aux Aaxa dans leur ensemble (le groupe des "autochtones»). En ce sens, un aîné de clan possède une forme de pouvoir sur les siens - pouvoir " public de second ordre », dirait Habermas - que l'on qualifierait de privé au regard de celui de l'autochtone, mais qui est surtout indissociable de ce dernier puisque relevant d'un "même pouvoir homogène " (Habermas 1978 : 16-17) et d'une même logique de primogéniture ou primorésidence. Ce type de hiérarchie englobante confere à l'autorité du chef peu de sens sans ses sujets : il leur est lié et doit se méfier du pouvoir de leur contestation.

Cela n'exclut pas la distinction entre les clans de rang différent, visible à certains signes : évitement du regard, prosternation de la part des sujets ainsi que marques d'allégeance et, particularité locale, travaux dans les champs du chef. De même, il ne faut pas négliger le fait que les sujets ont peu de poids dans les prises de décisions politiques collectives. En ce qui concerne la période antérieure à la formation des chefferies administratives, certains de mes interlocuteurs évoquent la seule autorité des autochtones avec leurs porte-parole ${ }^{18}$. Les observations des premiers administrateurs font cependant état de débats plus ouverts - même s'ils n'étaient pas forcément égalitaires ${ }^{19}$. À l'heure actuelle, dans le cadre de la

18. «Avant la colonisation ce n'était pas comme ça. Avant, il n’y a que les chefs coutumiers qui décidaient (autochtones). Maintenant c'est: "voter!" Il y avait le chef coutumier et les kwara abwatù [sujets qui s'abaissent]. En bas : les autochtones. Les trois, ensemble. Les apèrè-tèpe [porteparole] parlaient pour tous. C'est le facteur de la parole» (Crépin Diaiké, Kanak de Canala, 1996). 19. Dans un texte publié en 1869 , l'administrateur Ulysse de La Hautière notait à plusieurs reprises, à la suite d'observations faites à Canala au début des années 1860 (soit moins de dix ans après la prise de possession de l'archipel par la France), qu'un "conseil des chefs " regroupait des « orateurs », des «chefs de guerre», des «sorciers » et des « vénérables». 
chefferie d'Emma comme dans nombre d'autres petites chefferies ayant adopté l'institution coloniale du conseil des anciens, chaque clan et chaque lignage sont représentés au conseil siégeant dans un lieu nommé la «maison commune» soit par leur aîné, soit par un membre choisi dans chaque groupe. Le consensus lors des débats est généralement trouvé autour de la position adoptée par les membres des clans de haut rang, après qu'ils ont pris soin d'écouter tous les autres ${ }^{20}$. Les Aaxa tranchent donc dans des discussions concernant les affaires publiques tout en ne pouvant se passer des Kwara. Charge au kwara bwatù ("porte-parole") d'exposer leurs décisions au nom du chef. Ce sont donc littéralement "ceux qui parlent" ( $a x a)$ qui ont, sinon un pouvoir, du moins une autorité. L'exécution de leurs décisions ne se fait pas au nom de tous, comme dans un système démocratique, mais en leur nom propre. La pratique politique conforte ainsi le modèle hiérarchique et son assise symbolique : le chef incarne le politique - il est le politique -, mais un politique qui suppose une continuité entre les divers groupes qui le composent.

\section{Communs et particuliers}

Ce sont ces difficultés à ranger nettement les clans sujets du côté de la sphère privée et à désigner ceux de la chefferie comme représentant un espace pleinement public qui m'ont incitée à aller voir du côté des catégories de commun et de particulier utilisées par Habermas au sujet de l’organisation féodale "structurée par la représentation ». Bien que ni l'organisation foncière, ni les logiques de distinction hiérarchique, ni même la nature du lien de dépendance ne soient comparables, ce qui se joue dans le contexte féodal décrit peut néanmoins favoriser la compréhension de la structuration de l'espace public dans le monde kanak. Comme dans ce dernier, dans le modèle féodal évoqué ceux que l'on nomme les "autorités supérieures» (princes et État par exemple) «sont» le pays et ne le représentent pas. Dans ce cadre, la catégorie "public» ne peut évoquer de sphère sociale en tant que domaine de la pratique séparé21. En revanche, un statut de haut rang est représenté publiquement par celui

20. Cf. les procédures de la palabre africaine pour obtenir le consensus rapportées par Emmanuel Terray (1988).

21. Plus en détail, Habermas écrit : «D’un point de vue sociologique, c'est-à-dire à l'aide de critères propres à l'analyse institutionnelle, la sphère publique ne saurait apparaitre au sein de la société féodale du Moyen Âge comme un domaine propre, séparé d'une sphère privée [...]. Cette sphère publique structurée par la représentation ne se constitue pas tel un domaine social, comme la sphère de ce qui est public; au contraire, si l'on peut encore y appliquer ce terme c'est qu'elle joue, à peu de choses près, le rôle d'être signe caractéristique d'un statut. Le statut d'un seigneur quel que soit son rang, est en soi neutre au regard des catégories "public" et "privé"; mais celui qui en est le dépositaire représente ce statut publiquement : il se désigne et se présente comme l'incarnation d'une autorité comme toujours "supérieure" (1978 : 19). 
qui en est dépositaire parce que « quelque chose de bas ne peut être représenté ». Sont associés à cette autorité des privilèges et des droits particuliers dont ne disposent pas les "autorités inférieures». Ces dernières désignent l' "homme du commun" dépourvu de la "particularité " que serait un quelconque pouvoir de donner des ordres et qu'on comprendra ensuite comme pouvoir "public" (Habermas 1978 : 18). Le commun est donc l'homme ordinaire, le particulier, celui qui possède des privilèges. Si à ce dernier peut être attachée la notion de public, c'est dans le sens limité de l'incarnation d'un statut élevé qui lui confere une certaine autorité, indissociable cependant de celle des autorités inférieures. Les catégories de commun et de particulier comportent donc l'avantage indéniable de rendre mieux compte d'un modèle politique qui à la fois distingue et relie des groupes au sein de l'espace public. Je montrerai plus loin comment ce modèle s'illustre aussi spatialement. Au regard de l'ensemble des terres de la chefferie, la chefferie proprement dite (l'endroit où réside le chef) est, en effet, le lieu par excellence des particuliers, un lieu sacré car lieu de l'ancêtre. Mais là où se trouve la place "publique " (xwâjööchaa) et où se situe aujourd'hui la "maison commune" (lieu présenté comme étant l'«endroit où l'on s'amuse " tout autant que le lieu du débat politique) s'avère un lieu public dévolu surtout aux communs.

À ce type de logique d'organisation et d'exclusion politique correspond une pratique du secret précise. Il me semble intéressant de l'étudier afin de mieux comprendre comment s'opèrent clivages et continuités entre les groupes, et de mieux définir le rôle joué aussi bien par les Aaxa que par les Kwara dans les mécanismes politiques. Je vais commencer par expliciter les secrets aux fondements des principes politiques évoqués jusqu'ici (segmentation, distinction statutaire et complémentarité entre clans), avant de rendre compte du rôle joué par le secret dans la dynamique hiérarchique.

\section{Des secrets aux fondements de l'organisation politique \\ Les secrets claniques (xwâânîmö)}

Des récits privatifs?

Je l'ai déjà signalé, les histoires qui donnent aux unités de filiation leur identité sociale (xwâânîmö) doivent être transmises au sein de la "famille» (xwâjôösé, lignée) ou du lignage (xwâmwââdö). Autrefois cela se faisait de préférence le soir, autour du feu. Rares sont ceux dans un groupe à accéder à ce savoir jamais divulgué d'un bloc. Cela concerne souvent des aînés (plutôt des hommes et quelquefois des femmes), mais touche aussi des individus chez qui parents et grands-parents ont pu remarquer une capacité d'attention et de 
mémorisation importante, un goût pour ce type de connaissance ou encore une aptitude à garder le silence. Ces récits, parce qu'ils font l'objet d'une transmission entre soi, constituent des secrets ${ }^{22}$. L'exclusion qu'ils produisent participe du caractère privatif de ces unités sociales considérées comme des " groupes de parenté " grâce à un nom, des terres et des alliés communs.

\section{Des récits semi-publics}

Pour autant, tous ces attributs insèrent aussi ces individus dans un groupe politique au sein duquel ils occupent rang et fonctions. Et si l'on s'appuie sur le fait que ce n'est que rarement la réalité historique (l'ancienneté réelle d'implantation) qui, pour les migrants, détermine l'acquisition de leur rang mais bel et bien une décision collective issue de débats menés par les $A a x a$, les unités agnatiques composant une chefferie peuvent d'autant mieux être envisagées comme une "fiction publique» pour reprendre les termes de Michel Naepels ${ }^{23}$. C'est à cette occasion que les secrets généalogiques, gardés généralement au sein des xwâmwââdö, sortent momentanément de l'ombre pour être agencés publiquement avec d'autres récits du même type afin de conférer une certaine cohérence à une organisation politique hiérarchique donnée.

L'insertion des unités de filiation au sein du nèaaxa rè colonial territorialisé (chefferie) n'efface pas tout à fait les appartenances politiques antérieures. Lorsque ces mêmes xwâânîmö sont dévoilés hors de l'espace de résidence - et sont alors envisagés dans leur profondeur historique -, ils peuvent aussi rattacher ces groupes de parenté à d'autres groupes politiques. Chaque individu peut, en remontant dans son histoire, en se référant à un niveau de segmentation différent, se considérer comme appartenant à un autre groupe que celui qu'on lui connaît aujourd'hui. Chaque parcours clanique est ainsi constitué des rencontres avec d'autres segments aux divers points d'installations. Cela suppose que certains pans de ces histoires généalogiques sont partagés par des groupes différents. Il y a adéquation entre les lieux de circulation de ces récits - par conséquent aussi lieux de circulation de secrets - et les limites (variables) des clans. Aussi, lorsque rupture du secret il y a, se révèle du même coup la labilité des appartenances à des groupes d'agnats comme à des entités politiques.

22. Certains de mes interlocuteurs disent que ce sont des "paroles dans le noir». L'un d'eux m'a expliqué un aphorisme local qui dit: "Il ne faut pas exposer à la lune et au soleil le ventre de la parole car elle n'aurait plus de pouvoir" (Luther Méwaènô, 1997).

23. Termes empruntés à un texte non publié, "Sur l'autonomie", proposé à la discussion dans le groupe de travail Penser le contemporain, sous la responsabilité d'Étienne Balibar et Catherine Colliot-Thélène (mai 1997). Je remercie l'auteur de m’avoir donné accès à cette communication, qui m’a été utile pour la rédaction du présent article. 
Ces secrets sortis du groupe qui les détient mettent en lumière les mécanismes de fusion et fission décrits par Evans-Pritchard au sujet des groupes segmentaires; ils soulignent la difficulté à cerner des appartenances familiales et politiques univoques et définitives. En ce sens, l'usage du secret, dans le jeu du montrer/cacher qui le caractérise, dessine aussi des clans moins groupes de parenté privés qu'éléments semi-publics d'un dispositif politique.

\section{Les secrets des Aaxa}

L'autochtonisation du chef: un secret qui distingue

Le processus qui transforme le chef accueilli en autochtone est entouré lui aussi d'un mystère qui n'est que partiel. C'est par lui en grande partie que s'opère la distinction entre nobles et sujets. La procédure publique est partout identique dans le monde kanak. Les plus anciens de l'espace de résidence (ici kwara aéé et bwatù) "font asseoir " (pè cuè) le chef (aaxa) en lui donnant des terres et une femme issue de leur clan. À Canala, comme partout ailleurs semble-t-il, cette intronisation s'accompagnait aussi des rituels d'anthropophagie. (J'ai pu entendre des propos allant dans ce sens au sujet d'ingestion de "poissons" par le chef, ces derniers étant souvent présentés comme des substituts de la chair humaine dans cet univers néocalédonien où les aliments carnés étaient quasi inexistants.) Ce sont ces rites anthropophages qui étaient tenus partiellement secrets. Dans l'état actuel de mes connaissances, je ne suis pas en mesure de les décrire. Je renvoie aux travaux d'Alban Bensa qui évoque, pour l'aire paicî, la "fabrique du chef » en ancêtre local de son vivant, et ce tout le temps qu'il occupe la position de aaxa (Bensa \& Goromido 1996). Apparemment, dans cette aire, ce dernier recevait pour cela une alimentation particulière : tubercules classés anciens, viandes et poissons réservés et, périodiquement, de la chair d'un homme de haut rang (du clan qui l'a accueilli) qu'il était seul à consommer. Connu de tous - le sacrifice avait lieu en public devant l'allée du chef -, l'acte anthropophage proprement dit se pratiquait à l'écart du groupe. Le chef mangeait son grand sujet à l'abri des regards, dans sa demeure. Il semble que cette ingestion ait été considérée " comme une médication chargée de développer la puissance du chef ». Le foie et le cœur étaient déposés sur un lieu sacré "d'où le chef tirait des ancêtres la force et la réussite des siens dans les combats ». Mais ces actes de cannibalisme répétés auraient surtout permis de «faire du chef un ancêtre parmi les hommes». De cette transformation, les sujets ne devaient plus faire mention, à moins qu'ils ne veuillent délibérément jeter le discrédit sur le chef en contestant son ancienneté. 
À Canala, seuls les clans autochtones pouvaient apparemment évoquer ces actes. Un mythe (kêrê faxwara ou tèpe) qui appartient à ces clans anciens et qui décrit justement l'accueil et l'installation du chef ferait état de cette procédure de façon détournée. La première séquence du mythe évoque l'arrivée d'un étranger (parfois, selon les versions, avec un fils) qui réclame du feu aux autochtones. La deuxième séquence décrit le don que cet étranger leur fait après l'obtention de ce feu : il s'agit d'"oiseaux lunettes" (pî̀înî̀) grillés ${ }^{24}$. La troisième séquence mentionne un contre don qui prend la forme, selon les cas, d'une tarodière et d'une femme offerte en mariage à l'enfant ou seulement d'une femme donnée à l'étranger. Le mythe fait, selon toute vraisemblance, allusion, outre à une installation sur une terre et à la création de liens d'alliance, à une séance d'anthropophagie. Mon interprétation s'appuie sur les indications de mes interlocuteurs qui restent - non sans malice - flous sur la traduction de son titre. Ce récit s'intitule, en effet, soit dì rè pî̀m l'oiseau-lunette") soit duu rè pî̀mî̂̀ (la "domestication de l'oiseaulunette»). Dans les deux cas, l'oiseau est présenté comme l'équivalent symbolique de l'accueilli qu'on installe pour chef. Dans le premier cas, insister sur la rétribution de l'étranger laisse sous-entendre la dépendance du chef à l'égard des autochtones: celui-ci leur donne en quelque sorte son corps en échange de la chefferie. Dans le second cas, domestiquer l'étranger revient à dire qu'il a été transformé en une personne du terroir. Tout se passe comme si son ingestion par les autochtones du lieu l'assimilait à ces derniers (selon un schéma inverse à celui décrit par Bensa qui évoque cependant non l'intronisation mais la durée du règne du chef) ${ }^{25}$.

Si ces suppositions sont fondées, l'anthropophagie apparaît comme un élément ayant conduit à distinguer le chef (aaxa) de ses sujets en le plaçant du côté des gens de haut rang avec les grands sujets (kwara aéé et bwatù - les Aaxa, au sens générique. Par ce rite, il pouvait devenir un ancêtre vivant parmi les hommes, antérieur même aux fondateurs de l'espace de résidence.

En soustrayant une partie de l'acte anthropophage - et/ou en gardant pour eux le mythe de fondation de la chefferie -, les autochtones protège(ai)ent le chef de la contestation, grâce au pouvoir que confere le mystère. Si, de temps à autre, ils avaient intérêt - et l'ont toujours -

24. Zosterops xanthochroa: oiseau de la famile des Zostéropidés, considéré comme très commun en Nouvelle-Calédonie.

25. Dans l'aire linguistique paicî, Bensa (2000: 170) explique que l'investiture est pensée comme une capture. Le chef est présenté comme étant une pierre, une fougère, un coquillage, aux caractéristiques extraordinaires, découvert dans la forêt ou sur un rivage, puis rapporté à la maison et choyé ; cette trouvaille se transformera ensuite en un être humain à qui le pays sera confié. 
à montrer que ce dernier leur doit allégeance, masquer des éléments de la conversion de l'étranger en "ancien" de l'espace de résidence leur permet(tait) de mieux asseoir le chef - en le distinguant - devant des sujets qui ne peuvent se reconnaitre de cette unité politique qu'à la condition de croire à cette conversion, étant supposés être des descendants de cet aîné/autochtone.

La fixation des hiérarchies locales : un secret qui relie

L'autre secret qui contribue à structurer l'espace public kanak vise justement à établir une certaine continuité entre des Aaxa et des Kwara séparés statutairement par leur distance aux ancêtres fondateurs du terroir. Dans les petites et grandes chefferies de Canala, le personnage occupant la fonction de porte-parole du chef (apèrè-tèpe) - incluant un rôle d' «ambassadeur ", de "faiseur de paix» - m'a été présenté aussi comme celui en charge de "relier tous les clans entre eux".

À Emma (Amââ), lieu principal de mes enquêtes, on explique, dans un clan rattaché à celui qui occupe cette fonction aujourd'hui, que lors de la fondation de la chefferie actuelle, le porte-parole accomplit des gestes rituels dans un bosquet abrité des regards ${ }^{26}$. Ces rites auraient validé, pour chacun des clans et lignages, titres et rôles correspondant aux périodes et/ou conditions de leur installation. On dit que le porte-parole ordonna sur ce tertre les clans "par paliers». Cela fait directement référence à la structure inégalitaire du politique mais aussi à une certaine fixation des positions statutaires. Les paroles et gestes secrets du porte-parole se veulent en effet la garantie d'une certaine stabilité hiérarchique et, par conséquent, celle de l'harmonie entre les clans. Si le kwara bwatù, le cadet des autochtones, est en mesure d'opérer une telle chose, c'est parce qu'il est le seul, dans une chefferie, à avoir pris connaissance de l'ensemble des récits généalogiques des nouveaux arrivants et à savoir comment ils ont été ensuite intégrés à la chefferie. Fort de ce secret, il peut œuvrer à faire coïncider au mieux les anciennes et nouvelles positions statutaires. Le travail rituel secret du porte-parole participe donc à la création d'une interdépendance entre toutes les unités de filiation, sous la forme particulière d'un emboîtement des plus au moins nobles, en référence à l'ancienneté à laquelle chacun peut prétendre.

En reliant les clans les uns aux autres, le porte-parole établit un continuum qui signifie intégration de chaque partie à la totalité politique par succession de droits fonciers emboîtés et complémentarité de pouvoirs attribués. Le chef, par l'opération du rite anthropophage qui en faisait 
l'ancêtre du groupe (puis, peut-être aujourd'hui par l'ingestion d'équivalents symboliques carnés ?), devient quant à lui littéralement ce "tout " politique. La notion d'englobement est bien ce qui exprime le mieux ce principe politique hiérarchique. C'est pourquoi, on peut reprendre les termes de Habermas appliqués à un certain monde féodal pour désigner - dans ce système aussi - des "autorités supérieures " et d'autres «inférieures " participant toutes d'un "pouvoir homogène » et donc de l'espace public à des degrés divers.

\section{L'inscription d'un modèle dans l'espace}

Le lieu où opère le porte-parole se situe sur un tertre, derrière l'endroit où se rassemblent aujourd'hui les clans pour des réunions au sein de la "maison commune", sur la place dite "commune" (xwâjööchaa) ${ }^{27}$. Ce terme français manifeste le passage des missionnaires et leur tentative de faire de l'espace politique kanak hiérarchisé un espace communautaire où ce qui est commun est ce qui est public; mais on peut aussi entendre ce terme en référence à un modèle antérieur, "structuré par la représentation » comme le modèle féodal, où est commun ce qui renvoie plutôt au privatif (aux clans dépourvus de la possibilité de représenter le politique par défaut de statut élevé). La xwâjööchaa (l'«allée ou place de la maison commune») est en effet supposée être le lieu où sont inscrits de façon invisible « tous les clans par palier ${ }^{28}$. Mais cette place est encadrée à une extrémité par le tertre rituel de l'apèrè-tèpe ("porte-parole») et, à l'autre, par le tertre où vit le chef, à quelque trois cents mètres de là, non loin de la chapelle catholique du village ${ }^{29}$. Aussi la distinction demeure-t-elle entre ce lieu des communs et celui des particuliers. Ce qui les réunit plus sûrement c'est leur référence partagée aux ancêtres. Les trois tertres cités s’inscrivent, dans cette logique, sur les terres d'un clan désigné comme ancien. Les sujets sont littéralement entourés par le tertre du chef et par celui du porte-parole, et l'ensemble de cette unité politique est inscrit spatialement au sein d'un espace appartenant à des plus "anciens» encore. Ce dispositif spatial qui ne se donne pas à voir facilement me semble cependant très éclairant quant à ce que scellent les deux secrets politiques évoqués jusqu'ici : d'un côté la distinction statutaire et la hiérarchisation entre des clans de rangs chef et sujet, et, de l'autre, l'inclusion, voire l'emboîtement, des clans.

27. Xwâ désigne le "commencement". Jöö signifie la "corbeille du toit de la case", où sont conservés les éléments magico-religieux hérités des ancêtres d'une maisonnée; c'est aussi l'endroit où se relient tous les poteaux d'une case. Chaa veut dire "ensemble".

28. Explication émanant toujours de membres de clans "porte-parole».

29. C'est le signe matériel de la transformation de la chefferie avec l'évangélisation : l'ancestralité n'est plus la référence suprême unique ; être proche du dieu des Blancs contribue aussi à conférer au chef son caractère distinctif. 
À y regarder de plus près, ce dispositif souligne aussi une chose fondamentale dans le fonctionnement politique kanak aussi bien à Canala qu'ailleurs. En effet, le clan "englobant" les trois tertres dont il vient d'être question n'est pas le clan considéré actuellement à Emma comme le grand sujet, soutien du chef contemporain, mais comme un clan qui fut, dit-on, antérieurement "chef" (selon la traduction locale), sans que l'on puisse discerner avec certitude s'il s'agit du clan d'un ancien chef (aaxa) ou celui des anciens grands sujets (kwara aéé). C'est là le signe que, même dans l'espace, la mémoire historique du politique est conservée - et ce même si les clans qui prévalaient autrefois sont soumis à la hiérarchie créée lors d'une nouvelle configuration.

Des chefferies menacées par la rupture du secret

Aussi, malgré les rites des autochtones et du porte-parole, une configuration politique n'est jamais définitive, ni même totalement exclusive d'une plus ancienne. Ce qui permet de s'y tenir sur une durée plus ou moins importante, c'est le maintien du secret sur le passé. Personne n'ignore vraiment que les chefferies actuelles n'ont pas toujours eu le même visage, pas plus que les sujets (Kwara) n'ignorent que les rites des "chefs" (Aaxa) ont consacré un nouvel ordre social (c'est même la connaissance de leur existence qui confere un sens et une force à ce qu'ils consacrent, en même temps que de cacher les gestes effectués renforce leur poids). En somme, savoir que l'on classe les clans et qu'on les relie est nécessaire; le tout est de ne pas savoir comment. Il importe ensuite de ne plus faire mention ni des positions statutaires antérieures ni de ces rites (à moins de vouloir ouvertement décrier le «travail» des Aaxa). Ce silence collectif sur les généalogies et, de manière générale, sur tout ce qui concerne l'organisation sociale d'une chefferie à un moment donné, est nommé xwâfä ${ }^{30}$. Ce terme n'est pas traduit en français par «secret", bien que cette traduction semble être la plus proche de cette notion en langue xârâcùù. C'est, dit-on, une " limite sacrée ", un "mur ", au-delà duquel on ne peut remonter sans risquer de s'attirer des ennuis. Comme tout secret, il s'agit donc là encore d'une chose cachée dont on connaît l'existence sans pour autant en connaître forcément le contenu.

Mais c'est précisément le maintien, au-delà de ces xwâfä̈, d'une connaissance historique propre aussi bien aux clans (xwâmwââdö) qu'aux gens de haut rang dans les espaces de résidence (nè aaxa rè) qui permet, au sein des chefferies, de conserver une dynamique politique par ailleurs largement malmenée par le cantonnement et la "pacification». C'est par un jeu sur

30. De $x w a \hat{~ a u ~ s e n s ~ d e ~ " r e ́ s u l t a t ~ d ' u n e ~ a c t i o n ~ » ~ e t ~ d e ~} f a \ddot{a}$, «construire». 
les histoires généalogiques (xwâânîmö) et/ou sur les listes codées énumérant les clans appartenant à des grands clans antérieurs à la colonisation (xwââxa) que l'on tente aujourd'hui de subvertir, dans certaines limites, l'équilibre institué aux origines des chefferies coloniales ${ }^{31}$. Là encore le secret joue un rôle structurant puisque c'est à travers sa manipulation que des changements peuvent encore survenir ou au contraire être empêchés par l'énonciation de "vérités " venant conforter les positions établies. Plus exactement, la possibilité de dévoilement des secrets relatifs aux récits claniques et supraclaniques inscrit structurellement une dynamique politique dans le monde kanak; celle-ci ne vient pas pour autant contredire le modèle hiérarchique englobant.

\section{"Ouvrir" et "fermer" une parole ou le secret au service de la dynamique politique}

\section{Contestations et dévoilements}

La parole "mauvaise"

Ne pas évoquer sa généalogie revient à cautionner ce que les autochtones (kwara aéé et bwatù) d'un lieu ont un jour institué. En vertu des variantes de récits généalogiques circulant dans le secret des groupes agnatiques, et en vertu de la "fabrication " des positions occupées au sein d'une chefferie, l'énoncé de tout ou partie de la parole généalogique possède a contrario un caractère potentiellement conflictuel. Quelquefois elle vise à dévaloriser une personne avec laquelle on est par ailleurs en conflit. Il suffit par exemple de rappeler l'épisode d'une fuite après un viol ou un meurtre ou encore celui d'une ancienne défaite guerrière. Parfois, l'objectif du dévoilement des récits est plus vindicatif. Parler revient à vouloir imposer une version de son histoire au détriment d'une autre : c'est donc pouvoir prétendre au rang, aux titres et aux espaces fonciers de l'autre.

Les types de contestation qui peuvent se faire jour sont multiples. Les descendants de deux frères qui se sont séparés à un moment donné peuvent revendiquer l'histoire - et donc le statut - de celui qui a connu la position la plus avantageuse. Un accueilli peut tenter de se faire passer pour un accueillant en omettant de narrer la procédure d'accueil au profit de la seule mention du nom de la terre reçue. De même, un "clan » occupant un temps une position élevée, dépossédé de son rang par la force,

31. Au milieu du XXe siècle, l'établissement d'un État civil (1931) puis le premier recensement des Kanaks (1954) ont encore offert la possibilité à certains de s'octroyer des noms prestigieux qui n'avaient plus de porteurs. 
peut être tenté de rappeler qu'il a des droits sur les terres occupées aujourd'hui par son ennemi d'hier. Pour que ce type de contestation fonctionne il faut que l'adversaire n'ait pas les moyens de défendre ses droits, soit parce que son histoire s'est perdue au sein de son groupe, soit parce qu'il est effectivement lui-même un usurpateur.

Dans un contexte social où le respect est le maître mot du comportement idéal (Naepels 1998: 185-186), l'évitement physique et le silence sur ces questions généalogiques font donc partie de ce qui rend possible la vie en commun. Le secret gardé sur les xwâânîmö favorise - ou maintient - des relations sociales acceptables parce qu'il ne fige pas les identités et prémunit ainsi chacun de commettre un impair. Même dans le cadre de la transmission au sein de la maisonnée (xwâjööséé), il est parfois préférable de se taire, pour préserver l'honneur des siens en ne les initiant pas aux faits répréhensibles de leurs ancêtres. Ce qui était secret tombe alors peu à peu dans l'oubli sauf si d'autres, extérieurs au groupe, révèlent un jour ces secrets.

L'illusion du bénéfice de la parole définitive

La politique de rétrocession des terres spoliées par les colons depuis les années 1970 a favorisé la libération de la parole généalogique au niveau des clans comme des chefferies. Pour faire valoir ses droits auprès des administrations compétentes, il fallait pouvoir produire un récit univoque bien que collectif là où prévalent des droits emboîtés (Naepels 2006). Cela a donné lieu à des débats houleux autour de la recherche des "vrais" propriétaires. Dans certaines chefferies, dont celle d'Emma, la tentation de fixer définitivement les statuts dans l'espoir de faire taire les conflits à jamais est apparue. Mais pas plus ici qu'ailleurs ce ne fut un succès. Comment mettre d'accord publiquement des clans qui peuvent revendiquer des positions multiples? Quel moment de l'histoire choisir pour déterminer les statuts?

En définitive, cette option apparait bien comme une véritable transgression à la logique politique kanake qui nécessite une part de secret. Paradoxalement, c'est l'instabilité des statuts - le flou relatif maintenu qui permet la stabilité politique. Les Aaxa n'ont pas le pouvoir de proposer durablement et au grand jour une histoire univoque de leur chefferie. Ils peuvent seulement établir (sous le sceau d'un rituel secret de fondation) un ordre provisoire. C'est à cette condition que les sujets ne cherchent pas à quitter la chefferie comme ils pourraient le faire faute d'être suffisamment respectés. Le secret est donc fondamental au fonctionnement le plus harmonieux possible d'une chefferie dans la mesure où l'ordre établi à la fondation n'est alors ni ostentatoire ni définitif. 


\section{Les secrets sous la parole : garants de la paix}

\section{La médiation de l'apèrè-tèpe}

En cas de conflit ouvert au sein d'une chefferie, une parole doit pouvoir ramener la paix entre les parties qui s'opposent. L'histoire qui est alors narrée ne dessinera une fois encore qu'un paysage politique temporaire parce qu'elle saura aussi utiliser à bon escient le secret ${ }^{32}$. À Canala, celui qui jouera préférentiellement ce rôle de médiateur est bien évidemment le porte-parole (apèrè-tèpe), capable, par sa connaissance de l'ensemble des généalogies claniques (du moins de certaines versions d'entre elles), de trouver à ménager chacun dans ses susceptibilités (en mettant des éléments généalogiques en valeur et d'autres dans l'ombre) tout en affirmant ce qui justifie les positions occupées. Son pouvoir de «faiseur de paix» lui est donc conféré par son savoir acquis au bénéfice de son statut d'autochtone. C'est sa spécialisation historique qui en fait la clé de voûte de l'équilibre d'une chefferie ${ }^{33}$. Un bon porte-parole sait jouer sur l'éclairage qu'il donne aux faits et sait les combiner au mieux. Ce qu'il dit procède donc différemment de la "parole mauvaise" qui affirme simplement une position au détriment d'une autre.

\section{Les secrets des xwâjööchaa ("allées communes")}

Les mariages et les cérémonies de deuils - qui réunissent parents et affins, voire l'ensemble du groupe local (et même au-delà) - font partie des événements importants de la vie publique. Ce sont des moments de réaffirmation de l'ordre en vigueur comme de tensions ou de résolution des conflits éclatés en d'autres occasions. Des narrations généalogiques y sont énoncées à haute voix devant les clans rassemblés, respectivement sur les terres du mari et sur celles du mort (si c'est un homme) ou, dans les deux cas, sur celles de son mari (s'il s'agit d'une femme). Un ordre de prise de parole sera respecté en fonction du rang des groupes présents. Pour ceux qui reçoivent, il s'agit de magnifier les alliés, de faire valoir la force des liens qui les unissent tous. A minima, il faut faire en sorte de respecter chacun des parents.

32. À l'échelle des grandes chefferies regroupant plusieurs chefferies, d'autres types de narrations que les xwâânimö sont aussi convoquées: il s'agit des xwââxa ("origine de la parole»), listes de noms correspondant à la hiérarchie d'un grand clan, d'où sont évacués les événements historiques, les conditions de déplacements des clans et celles de leur accueil. Ces listes sont déclamées dans un ordre étudié, en fonction du contexte d'énonciation. Elles sont parfois agrémentées de toponymes ou de citations des symboles attachés aux groupes.

33. On peut s'interroger sur ce qu'implique la détention de tant de secrets par un seul homme sur la spécificité de l'organisation politique hiérarchique à Canala. Est-ce le premier pas vers la constitution d'un ordre politique plus stable - proche de celui qui pourrait être produit par écrit - dans la mesure où ce personnage est capable, plus que quiconque, de produire s'il le veut une histoire globale et univoque? Est-ce là l'image d'une centralisation de l'autorité plus forte qu'ailleurs en pays kanak? 
Moins que jamais la parole n'est ici libre. L'énonciation des généalogies revient à la production, dans le même temps que d'un récit politique, d'un secret public au double sens de "secret au contenu politique» (comme ceux déjà évoqués jusqu'ici), mais aussi au sens de "secret élaboré en public». Derrière chaque discours se tient une parole cachée, qui reste privée, mais dont chaque membre des clans présents sur l'allée cérémonielle (xwâjööchaa) peut supposer l'existence sans bien en connaître le contenu. On taira généralement tout ce qui a trait aux modalités de l'accueil (abandon, fusion, création de noms) ${ }^{34}$. On dit qu'on laisse "fermé (mwêrê) un récit ", qu'on consolide le "mur " (xwâfä) construit par le passé sur tout ce qui précède. "Fermer» des éléments du récit revient, à l'instar des vertus déjà attribuées ici au secret, à donner au maximum le sentiment que la présence et la place de chaque clan au sein d'un groupe local ne sont pas le fruit d'une «fabrication" par les autochtones.

Ainsi, par ce type de parole qui énonce autant qu'elle cache, la hiérarchie s'en trouve renforcée. Ce qui a été construit à l'aide de secrets est présenté non pas comme une fiction mais comme le fruit d'une réalité historique où chacun occupe effectivement une place en fonction de son ordre d'arrivée réel. L'actualisation des paroles généralement cachées renvoie aux secrets de fondation et permet d'en perpétuer l'efficacité tout en réajustant les positions en fonction de nouveaux rapports de force qui pourraient se faire jour (numérique notamment). Mais si les individus peuvent éventuellement changer de statut, le modèle statutaire, lui, ne s'en trouve pas affecté. L'existence de deux rangs séparés, entre lesquels existent une certaine continuité politique ainsi que leur englobement par le chef, ancien entre les Anciens, demeure. J'espère avoir ainsi montré - à l'aide d'une réflexion sur l'organisation politique et son usage du secret que les catégories privé/public sont inadéquates pour opposer chefferie et sujets. On obtient une image plus juste du modèle politique kanak en le considérant comme inégalitaire mais partagé à certains degrés entre personnes de haut rang - particulières - et personnes de rang moindre communes. C'est là une piste pour sortir des descriptions contradictoires véhiculées sur la chefferie kanake par les premiers observateurs étrangers

34. Dans la petite chefferie d'Emma à Canala, on raconte par exemple qu'on enterra l'ancien nom de la dernière rescapée d'une guerre (arrivant enceinte à la suite d'un viol) en creusant un «four kanak ", composé de pierres chaudes enfouies sous la terre, et qu'on le recouvrit de trois peaux de bananes. L'envers positif de cette histoire consiste à dire qu'en arrivant, la nouvelle venue, comme tout un chacun, a offert un présent à ses hôtes (moofädë), scellant ainsi son lien avec ses nouveaux parents qui lui ont redonné un nouveau nom. 
qui évoquent soit un chef tout puissant soit un chef sans pouvoir. C'est sans doute ceux qui s'attachaient à l'idée d'incarnation qui penchaient pour la privatisation de la chose publique, tandis que ceux qui repéraient la publicité relative des groupes segmentaires tendaient à supposer que les gens de haut rang étaient sans ressource face à leur sujets. Utiliser les catégories «commun » et "particulier " permet donc de repenser la réalité de la pratique politique kanake. Pour autant, la délimitation d'une sphère privée et d'une sphère publique, pas plus que le repérage de communs et de particuliers, ne permettent de déduire systématiquement une pratique politique. Un modèle qui sépare nettement sphère privée et sphère publique (cette dernière représentant le groupe sur un plan égalitaire) n'exclut certes pas l'existence d'un espace public investi par ceux que le pouvoir est censé représenter, mais il n'exclut pas non plus la stigmatisation et/ou la marginalisation de certains groupes au sein de l'espace public. Un modèle qui distingue communs et particuliers, s'il n'équivaut pas à se trouver en présence d'un pouvoir confisqué par les gens de haut rang, ne met pas non plus à l'abri d'une telle possibilité. Seule l'analyse de la pratique du pouvoir au sein d'organisations concrètes permet de se prononcer, au-delà des modèles.

Institut de recherche interdisciplinaire sur les enjeux sociaux, Ehess, Paris Centre de recherche et de documentation sur l'Océanie, Ehess, Marseille cdemmer@cegetel.net

MOTS CLÉSIKEYWORDS : secret - chefferie/chieftaincies - modèle politique/political model dynamique politique/political process - privé/public/private/public - Kanaks - NouvelleCalédonie/ New Caledonia. 
Barrau, Jacques

1956 L'Agriculture vivrière autochtone de la Nouvelle-Calédonie. Précédée de L'Organisation sociale et coutumière de la population autochtone, par Jean Guiart. Nouméa, Commission du Pacifique Sud.

\section{Bensa, Alban}

1988 "Océanie : organisations, règles et pouvoir ", in L'Atlas des Religions. Paris, Encyclopaedia Universalis : 250-251.

1992 "Terre kanak : enjeu politique d'hier et d'aujourd'hui : esquisse d'un modèle comparatif ", Etudes rurales 127-128 : 107-132. 2000 «L'impossible héros : chefs et leaders en pays kanak, Nouvelle-Calédonie XIX'-XXe ", La Nouvelle Revue du Pacifique 1 (1) : 160-181.

\section{Bensa, Alban \& Antoine Goromido}

1996 "Lauto-sacrifice du chef dans les sociétés kanak d'autrefois ", in Maurice Godelier \& Jacques Hassoun, eds, Meurtre du père, sacrifice de la sexualité. Approches anthropologiques et psychanalytiques. ParisStrasbourg, Arcanes, Recherche psychanalytique : $103-120$.

1998 "Contraintes par corps: ordre politique et violence dans les sociétés kanak d'autrefois ", in Maurice Godelier \& Michel Panoff, eds, Le Corps humain. Supplicié, possédé, cannibalisé. Paris-Amsterdam, Éd. des Archives contemporaines : 169-197.

\section{Bensa, Alban \& Jean-Claude Rivierre}

1982 Les Chemins de l'alliance.

L'organisation sociale et ses représentations en Nouvelle-Calédonie, région de Touho, aire linguistique cèmuhî... Paris, SÉLAF («Langues et cultures du Pacifique»1).

\section{Dauphiné, Joël}

1989 Les Spoliations foncières en NouvelleCalédonie, 1853-1913. Paris, L'Harmattan. 1990 Canala et la France (1854-1863). Nouméa, Centre territorial de recherche et de documentation pédagogiques.

\section{Demmer, Christine}

2002 Les Héritiers d'Éloi Machoro

(1941-1985). Une génération nationaliste au pouvoir à Amââ et Kûoö, villages de Xârâcùu, Canala (Nouvelle-Calédonie). Paris, École des hautes études en sciences sociales, thèse de doctorat.

\section{Doumenge, Jean-Pierre}

1975 Paysans mélanésiens en pays Canala, Nouvelle-Calédonie. Talence, Centre d'études de géographie tropicale ("Travaux et documents de géographie tropicale»17).

\section{Guiart, Jean}

1962 «Dualisme et structure du contrôle social en pays Canala, Nouvelle-

Calédonie", L'Homme 2 (2) : 49-79.

\section{Habermas, Jürgen}

1978 L'Espace public. Archéologie de la publicité comme dimension constitutive de la société bourgeoise. Paris, Payot.

\section{Hocart, Arthur Maurice}

1978 Rois et Courtisans. Paris, Le Seuil.

\section{La Hautière, Ulysse de}

1869 Souvenirs de la Nouvelle-Calédonie.

Paris, Challamel aîné.

\section{Lenormand, Maurice- $\mathrm{H}$.}

1953 «L'évolution politique des autochtones de la Nouvelle-Calédonie ", Journal de la Société des Océanistes 9 : 245-299.

Merle, Isabelle

1998 "La construction d'un droit foncier colonial : de la propriété collective à la constitution des réserves en NouvelleCalédonie ", Enquête 7 : 97-126. 
Naepels, Michel

1998 Histoires de terres kanakes. Conflits fonciers et rapports sociaux dans la région de Houaïlou (Nouvelle-Calédonie). Paris, Belin.

2000 «Le conflit des interprétations. Récits de l'histoire et relations de pouvoir dans la région de Houaïlou (Nouvelle-Calédonie) ", in Bertrand Masquelier \& Jean-Louis Siran, eds, Pour une anthropologie de l'interlocution. Rhétoriques du quotidien. Paris-Montréal, L'Harmattan : 337-357.

2006 «Réforme foncière et propriété dans la région de Houaïlou (NouvelleCalédonie) », Études rurales 177 : 43-54.

\section{Salomon, Christine}

1998 «La personne et le genre au Centre Nord de la Grande Terre (NouvelleCalédonie)", Gradhiva 23 : 81-100.

\section{Saussol, Alain}

1979 L'Héritage. Essai sur le problème foncier mélanésien en Nouvelle-Calédonie. Paris, Société des Océanistes.

\section{Soriano, Éric}

2001 Une trajectoire du politique en Mélanésie. Construction identitaire et formation du personnel politique : l'exemple kanak de Nouvelle-Calédonie (1946-1999). Montpellier, Université Montpellier 1, thèse de doctorat.

\section{Terray, Emmanuel}

1988 «Le débat politique dans les royaumes de l'Afrique de l'Ouest: enjeux et formes ", Revue française de science politique 38 (5): 720-731.

\section{RÉSUMÉ/ABSTRACT}

Christine Demmer, Secrets et organisation politique kanake: pour sortir des catégories privélpublic. - Ce texte décrit l'utilisation du secret au sein des espaces de résidence kanaks (chefferies) pour mettre en lumière son rôle dans la structuration et la dynamique politiques, mais aussi pour interroger l'usage des catégories privé/public dans la définition de ces systèmes. L'on s'aperçoit que certains secrets sont au cœur de ce qui fonde le modèle politique: à savoir une distinction statutaire qui permet au chef d'incarner ses sujets et un continuum entre des unités segmentaires semi-publiques se référant à un même ancêtre fondateur. Cela établi revient à souligner l'inadéquation d'une coupure franche entre sphère privée et sphère publique. C'est pourquoi, cherchant à trouver d'autres termes plus adaptés, l'article montre que les catégories de particulier et de commun empruntées à Habermas peuvent s'appliquer pour qualifier la chefferie kanake.
Christine Demmer, Secrets and Kanak Political Organization: Moving Beyond the Categories of "Private" and "Public". - The use of secrets in Kanak residential areas (chiefdoms) is described to shed light on how secrecy shapes and impels politics; but questions are also raised about how the categories of "private» and "public" define these systems. Certain secrets lie at the core of the political system's foundation, namely the status distinction whereby a chief incarnates his subjects and personifies a continuum between semipublic lineage segments, which refer to a common ancestor. Developing this hypothesis leads to emphasizing the inadequacy of a clear-cut separation between the private and public spheres. In a quest to find more suitable terms, the categories of "particular" and "common ", borrowed from Habermas, are applied to Kanak chieftaincies. 